\title{
Clinical efficacy and safety of colistin treatment in patients with pulmonary infection caused by Pseudomonas aeruginosa or Acinetobacter baumannii: a meta-analysis
}

\author{
Huagen Zhang, Qianyun Zhang
}

Respiratory Medicine Meizhou People’s Hospital, Meizhou Guangdong, Meizhou, China

Submitted: 20 October 2013

Accepted: 9 January 2014

Arch Med Sci 2015; 11, 1: 34-42

DOI: $10.5114 /$ aoms.2015.48158

Copyright ๑ 2015 Termedia \& Banach

\section{Abstract}

Introduction: The aim of this study was to evaluate the efficacy and safety of colistin treatment in patients with pulmonary infection caused by Pseudomonas aeruginosa or Acinetobacter baumannii.

Material and methods: The relevant studies were identified through a search of public databases including PubMed, MEDLINE and EMBASE up to December 2012. A meta-analysis was conducted to compare the clinical response, mortality and renal damage of colistin (colistin group) versus other effective antibiotics (control group). The odds ratio $(O R)$ was chosen as the effect size. Results: A total of 9 studies were eventually identified. The result of the meta-analysis showed that the pooled $O R$ of clinical response was 1.24 $(95 \% \mathrm{Cl}=0.68-2.27, p>0.05)$ for patients in the colistin group versus the control group, indicating no significant difference in efficacy between colistin and control groups. Similar results were obtained by the further subgroup meta-analyses by sample size, research year, ethnicity and study method. Treatment with colistin versus other agents did not affect hospital mortality $(O R=1.05,95 \% \mathrm{Cl}=0.58-1.89, p>0.05)$ or renal damage $(O R=1.25$, $95 \% \mathrm{Cl}=0.78-2.00, p>0.05)$. The combined estimate of our analysis was strong across multiple sensitivity analyses and without significant publication bias.

Conclusions: Our results suggest that colistin may be as efficacious and safe as standard antibiotics for the treatment of pulmonary infection.

Key words: colistin, pulmonary infection, Pseudomonas aeruginosa, Acinetobacter baumannii, meta-analysis.

\section{Introduction}

Multidrug-resistant (MDR) gram-negative bacilli, mainly Pseudomonas aeruginosa (P. aeruginosa) and Acinetobacter baumannii (A. baumannii), are major nosocomial pathogens worldwide [1-3]. The emergence of infections caused by multidrug-resistant Gram-negative bacteria poses a great challenge for infection control [4-6].

Colistin, also called polymyxin E, is a polypeptide antibiotic. Colistin is commercially available as colistin sulfate (for oral and topical use) and colistimethate sodium (for parenteral use, intravenously and intramuscularly) [7]. Also, both forms can be administered by inhalation [8].

\author{
Corresponding author: \\ Huagen Zhang \\ Respiratory Medicine \\ Meizhou People's Hospita \\ Meijiang Distric \\ Huangtang Road No. 63 \\ Meizhou Guangdong, 514000 \\ Phone: +860753 2131903 \\ Fax: +8607532204840 \\ E-mail: zhanghuagenn@ \\ hotmail.com
}


Although colistin showed excellent activities against many species of gram-negative bacteria in vitro [9, 10], use of colistin has been limited due to limited clinical efficacy and significant nephrotoxicity and neurotoxicity. During the last 2 decades, colistin was mainly restricted to treat acute exacerbations of lung infections in patients with cystic fibrosis $[5,11,12]$. However, the increasing frequency of pulmonary infections and lack of new agents effective against the resistant strains have led to the reconsideration of colistin [8]. Some reports have demonstrated that modified dosage of colistin could be used as a therapeutic intervention for patients with pulmonary infections due to MDR $P$. aeruginosa and $A$. baumannii $[7,8,13,14]$.

Nevertheless, whether colistin in the treatment of Gram-negative bacteria infection is superior to other antibiotics is controversial [15-18]. Metaanalysis is a statistical procedure for combining the results of several studies to produce a single estimate of the major effect with enhanced precision [19-21], and it is considered as a powerful tool for summarizing inconsistent results from different studies. Hence, the aim of the present meta-analysis was to systematically evaluate the efficacy and safety of colistin in the treatment of pulmonary infection caused by $P$. aeruginosa or A. baumannii.

\section{Material and methods}

\section{Literature search}

Electronic databases including PubMed, MEDLINE, EMBASE, Springer, Elsevier Science Direct, Cochrane Library and Google Scholar were searched for all publications on colistin treatment in patients with pulmonary infection caused by $P$. aeruginosa or $A$. baumannii up to December 2012 without language restrictions. The main search terms were "colistin" OR "polymyxin" OR "polymyxin E" AND "pulmonary infection" OR "lung infection" AND "Pseudomonas aeruginosa" OR "Acinetobacter baumannii" OR "Gram-negative bacilli" AND "study" OR "trial”. Meanwhile, references from retrieved papers were checked for any additional studies. We only recruited data from the full published paper, not any meeting or conference abstract. Two investigators ( $\mathrm{HZ}$ and $\mathrm{QZ}$ ) independently searched the electronic databases.

\section{Inclusion and exclusion criteria}

Studies were included in the meta-analysis if they met the following criteria: 1) The investigations concerned patients with bronchiectasis (prospective studies, retrospective studies or cross-sectional studies, etc.); 2) The objects were the pulmonary infected patients (range of age was not limited); 3) The method of treatment was colistin against other relevant antibiotic treatment (ways of application of the drugs were not limited); 4) The effect size was the odds ratio. Sample size was not limited; 5) Studies were published in the English language. Studies were excluded if one of the following existed: 1 ) reduplicated studies or records; 2) review of literature; 3) no control design.

\section{Quality assessment and data extraction}

Quality was assessed using the factors mainly including the methods of studies, sample size, and recruitment of respondents. Initial screening was done by reading the document title and abstract. Secondary screening was conducted by reading the full text of papers. Finally, studies were included according to the inclusion and exclusion criteria. Data items included study details (such as the first author's name, research year of study, year of publication, location of participants, and method of studies), characteristics of participants (such as age and sample size), and Acute Physiological and Chronic Health Evaluation II (APACHE II) score. We contacted authors of the included studies to obtain further information about data items. We used standardized data extraction forms to record the first author's name, time of study, time of publication, country, geographic location, sample size, age, study method and events of the colistin group versus the control group. Quality assessment and data extraction were independently conducted by two investigators. Disagreements were resolved by discussion to come to an agreement.

\section{Statistical analysis}

Our primary analysis was focused on a comparison of the odds ratios (ORs) in the colistin treated versus non-colistin treated patients with pulmonary infection caused by $P$. aeruginosa or A. baumannii (colistin group vs. control group). Then, stratified analysis by sample size, research year, participants' geographic location and study method was also conducted.

The meta-analysis was performed using fixed or random effect models. The point estimates of the odds ratio $(O R)$ and its $95 \%$ confidence interval $(95 \% \mathrm{Cl})$ were estimated for each study. We assessed the within- and between-study variation or heterogeneity by testing Cochran's Q-statistic and ${ }^{2}$-statistic $[22,23]$. Values of $p<0.10$ or ${ }^{2}$ value $>50 \%$ was considered to be heterogeneous. When heterogeneity was detected across studies, the random effect model was used for meta-analysis as well as to take into account the possibility of heterogeneity between studies. Otherwise, the fixed effect model was used. The fixed effect model assumes that all of the studies are estimating the same underlying effect and considers 
4052 potentially relevant reports identified and screened (PubMed, 1206; MEDLINE, 783; Springer, 653; Elsevier Science Direct, 892; Cochrance Library, 55; Google Scholar, 463)

\section{$\downarrow$}

365 potentially relevant reports after duplicate removed

326 excluded by review of abstract (65 reviews; 168 not pulmonary infected; 93 not reported colistin data)

39 retrieved for detailed assessment

30 excluded by review of full text

(19 for just only reported colistin data but not for comparation;

11 due to not available data)

9 separate studies included in meta-analysis

Figure 1. Flow diagram for selection of studies and specific reasons for exclusion from the meta-analysis

only within-study variation. The overall or pooled estimate of ORs was obtained using the Mantel-Haenszel method in the fixed effect model [24] and using the DerSimonian and Laid method in the random effect model [25]. Pooled ORs in the meta-analysis were calculated by weighting individual $O R s$ by the inverse of their variance. The significance of the pooled ORs was determined by the Z-test.

A funnel plot was used to detect publication bias. An asymmetric plot infers a possible publication bias. The asymmetry of the funnel plot was further assessed using Egger's linear regression test [26], which measures funnel plot asymmetry on the natural logarithm scale of the effect size. Values of $p<0.05$ was considered as indicating statistically significant publication bias.

In order to test the reliability of the results, we also performed a sensitivity analysis after removal of the study with biggest or smallest sample size to repeat the meta-analysis.

Analyses were performed using the software Review Manager 5.1 (Cochrane Collaboration, http:// ims.cochrane.org/revman) and the STATA software package v.11.0 (Stata Corporation, College Station, TX, USA).

\section{Results}

\section{Characteristics of eligible studies}

There were 4052 papers potentially relevant to the search terms (PubMed: 1206; MEDLINE: 783; Springer: 653; Elsevier Science Direct: 892; Cochrane Library: 55; Google Scholar: 463). The study selection process is shown in Figure 1. There were 365 potentially relevant studies after duplicates were removed. After reviewing the title and abstract, 326 of these articles were excluded (65 were review articles; 168 did not concern pulmonary infection; 93 did not report colistin data). Af ter reviewing the full publication, 30 articles were excluded (18 for only reporting colistin data but not for comparison; 12 due to unavailable data).

Finally, 9 studies [1, 9, 16, 27-32] were included in this meta-analysis. The characteristics of the included studies are summarized in Table I. The included studies were published between 2003 and 2012. Of the 9 included studies, 4 were conducted in Europe, 3 in America, 1 in Asia and 1 in Africa. A total of 940 patients with pulmonary infection caused by $P$. aeruginosa or $A$. baumannii were considered in this meta-analysis. Among them, 411 were treated with colistin (colistin group) and 529 were treated with other antibiotics such as ampicillin, sulbactam, cefepime, meropenem piperacillin or tazobactam (control group). The patients' age was between 3 and 103 years old. Sample size varied between 13 and 130. These included studies were cohort studies or case-control studies. The mean value of the APACHE II score was between 14.0 and 22.3 .

\section{Meta-analysis of efficacy of colistin versus other antibiotics}

Overall meta-analysis as well as subgroup meta-analyses by sample size, research year, participants' geographic location (ethnicity) and study method were conducted to compare the efficacy of colistin against other antibiotics in treatment of pulmonary infection caused by $P$. aeruginosa or A. baumannii.

As shown in Table II and Figure 2, a total of 9 separate studies [1, 9, 16, 27-32], consisting of 940 patients (411 in the colistin group and 529 in the control group) were included in this meta-analysis. There was significant heterogeneity across the studies $\left(Q^{2}=29.9, R^{2}=73.2 \%\right.$, $p<0.01)$. The overall meta-analysis indicated that the pooled $O R$ was $1.24(95 \% \mathrm{Cl}=0.68-2.27$, $p>0.05)$ for patients in the colistin group versus patients in the control group. The funnel plot was not symmetrical obviously, indicating that publication bias may exist. However, the result of Egger's linear regression showed no publication bias in the studies included in this meta-analysis $(t=1.76$, $p>0.05)$.

The results of the further subgroup meta-analyses are shown in Table II. The pooled $O R$ was $0.75(95 \% \mathrm{Cl}=0.41-1.38, p>0.05)$ when considering 4 studies in which the sample size was more than or equal to 40, and the pooled OR was 2.15 (95\% Cl $=0.92-5.00, p>0.05)$ when considering 5 studies in which the sample size was less than 


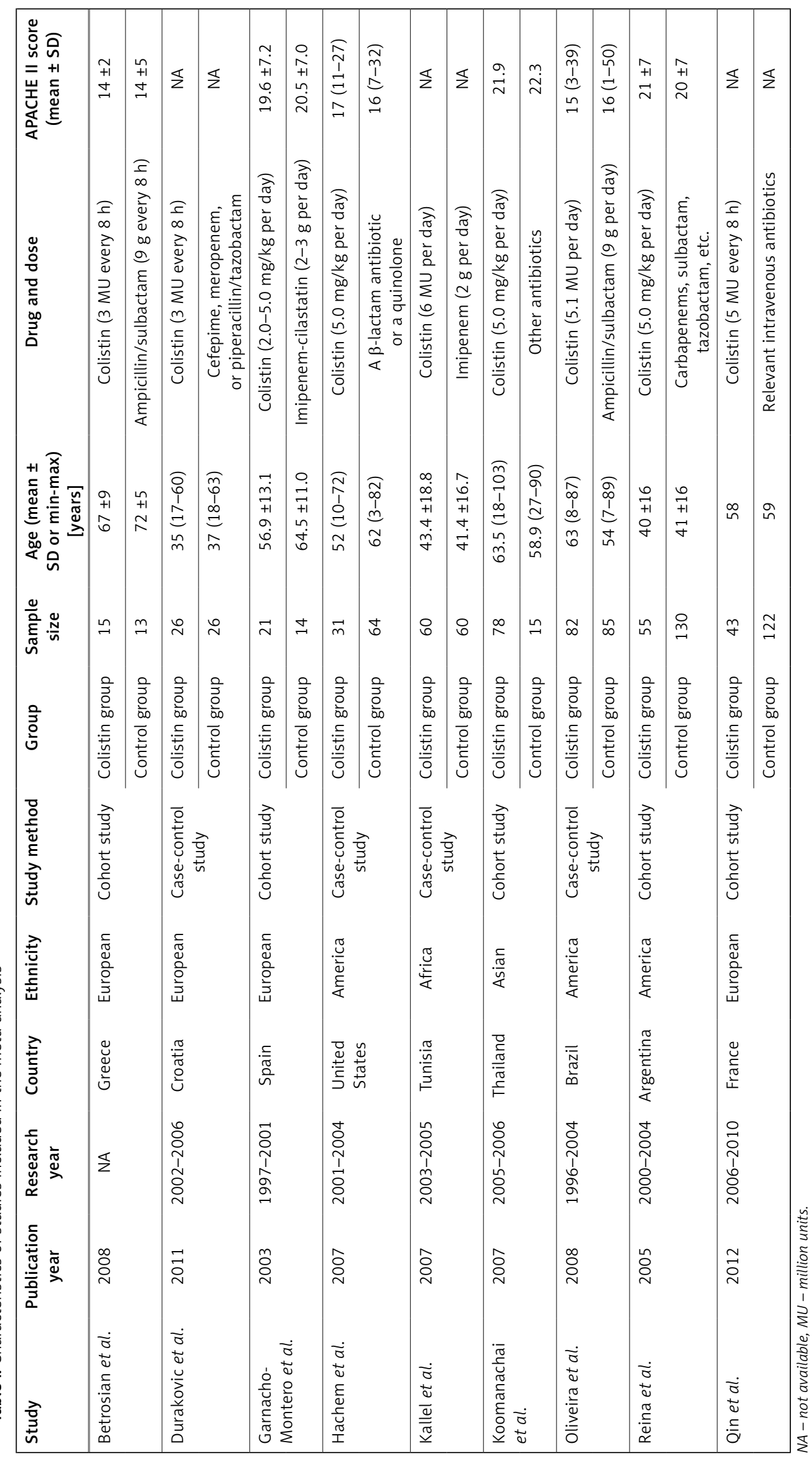




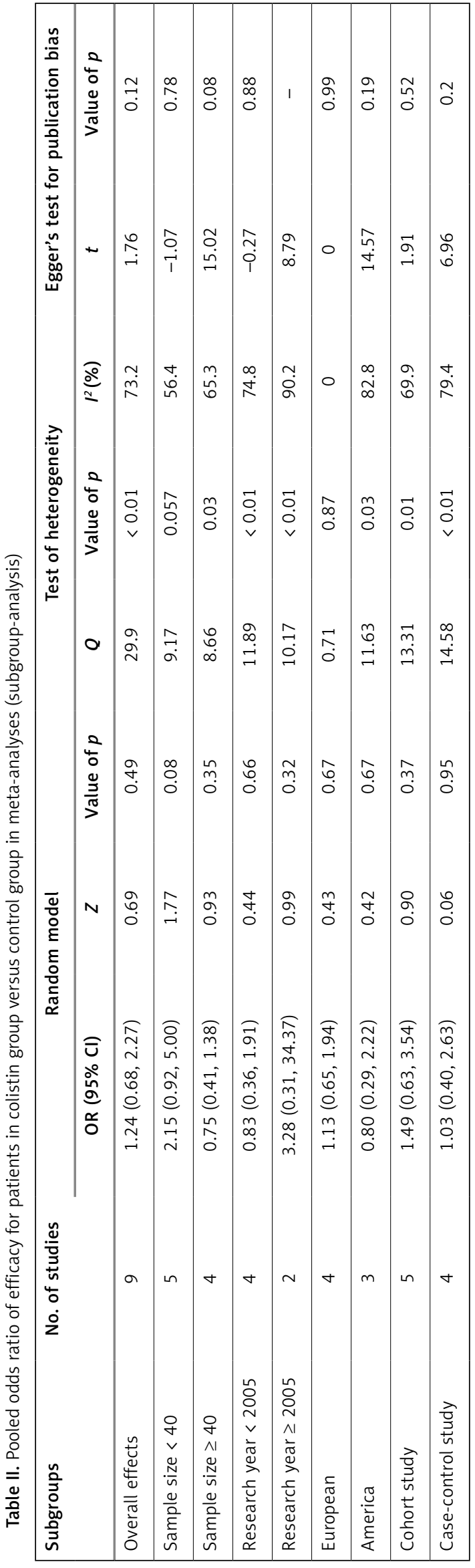

40. The pooled $O R$ was $3.28(95 \% \mathrm{Cl}=0.31-34.37$, $p>0.05)$ for studies published in or after 2005, and the pooled $O R$ was $0.83(95 \% \mathrm{Cl}=0.36-1.91$, $p>0.05)$ for studies published before 2005. The pooled $O R$ was $1.13(95 \% \mathrm{Cl}=0.65-1.94, p>0.05)$ for studies conducted in Europea and 0.80 $(95 \% \mathrm{Cl}=0.29-2.22, p>0.05)$ for studies conducted in America. The pooled ORs were $1.49(95 \% \mathrm{Cl}=$ $0.63-3.54, p>0.05)$ and $1.03(95 \% \mathrm{Cl}=0.40-2.63$, $p>0.05)$ for 5 cohort studies and 4 case-control studies, respectively.

No significant difference in efficacy between the colistin group and the control group was detected using overall and subgroup meta-analyses $(p>0.05)$.

\section{Meta-analysis of mortality of colistin versus other antibiotics}

As shown in Figure 3, a total of 7 separate studies $[1,9,16,27,29,31,32]$, consisting of 635 patients (296 in the colistin group and 339 in the control group) with pulmonary infection caused by $P$. aeruginosa or $A$. baumannii were included in this meta-analysis. Significant heterogeneity was detected between studies $\left(Q^{2}=12.0, R^{2}=50.0 \%\right.$, $p<0.1)$. The overall meta-analysis indicated that the pooled $O R$ was $1.05(95 \% \mathrm{Cl}=0.58-1.89$, $p>0.05)$ for patients in the colistin group versus patients in the control group. No significant difference in mortality was observed between the colistin group and the control group ( $p>0.05$ ). We also performed Egger's linear regression test. The result showed that there was no publication bias in the studies included in this meta-analysis $(t=1.54, p>0.05)$.

\section{Meta-analysis of renal damage of colistin versus other antibiotics}

As shown in Figure 4, a total of 6 separate studies [1, 9, 16, 27, 29, 32], consisting of 470 patients (253 in the colistin group and 217 in the control group) with pulmonary infection caused by $P$. aeruginosa or $A$. baumannii were included in this meta-analysis. No heterogeneity between studies was detected $\left(Q^{2}=8.22, I^{2}=39.0 \%, p>0.1\right)$. The overall meta-analysis indicated that the pooled OR was $1.25(95 \% \mathrm{Cl}=0.78-2.00, p>0.05)$ for patients in the colistin group versus patients in the control group. There was no significant difference in renal damage between the colistin group and the control group. No publication bias was detected in the studies included in this meta-analysis using Egger's linear regression $(t=1.49, p>0.05)$.

\section{Evaluation of sensitivity analysis}

We performed a sensitivity analysis by removing one study each time and rerunning the mod- 


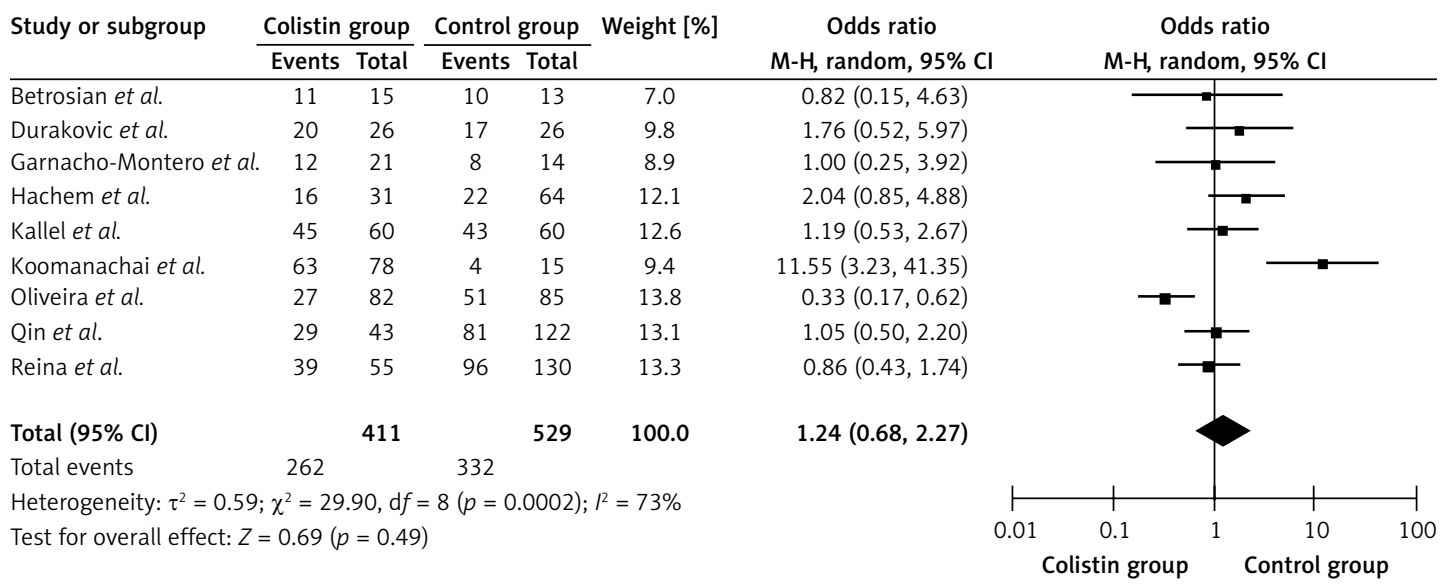

Figure 2. Forest plot of studies with efficacy of colistin group versus control group

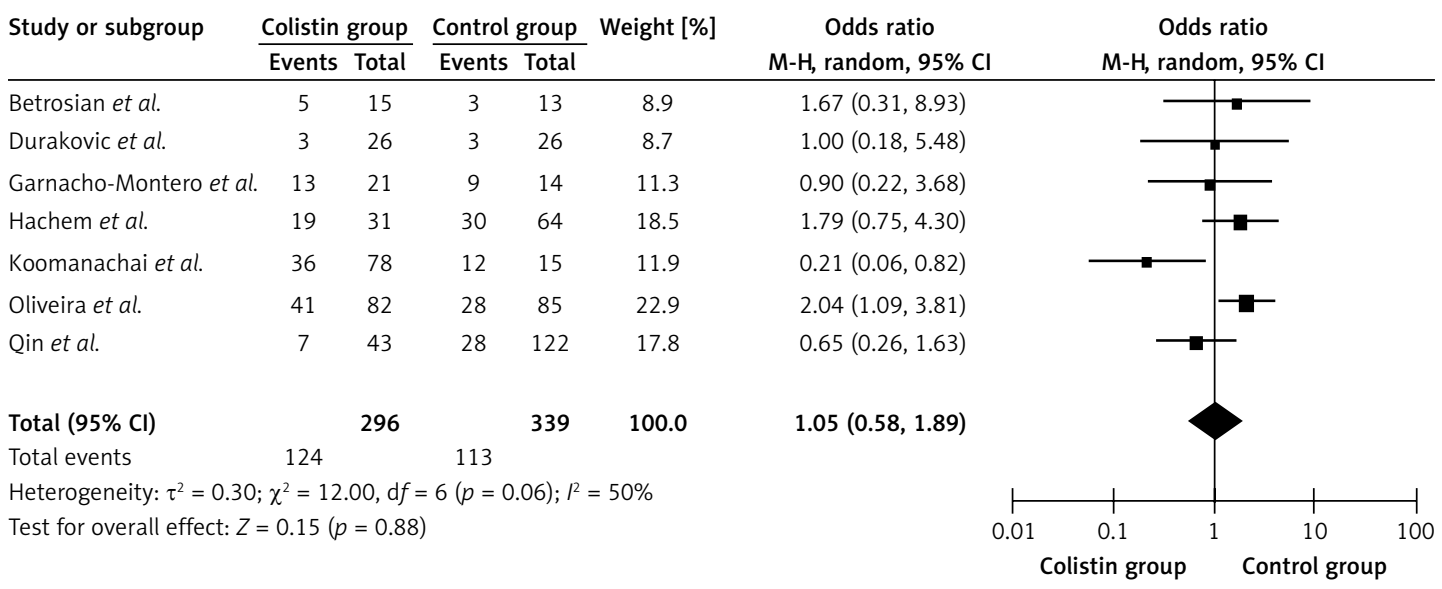

Figure 3. Forest plot of studies with mortality of colistin group versus control group

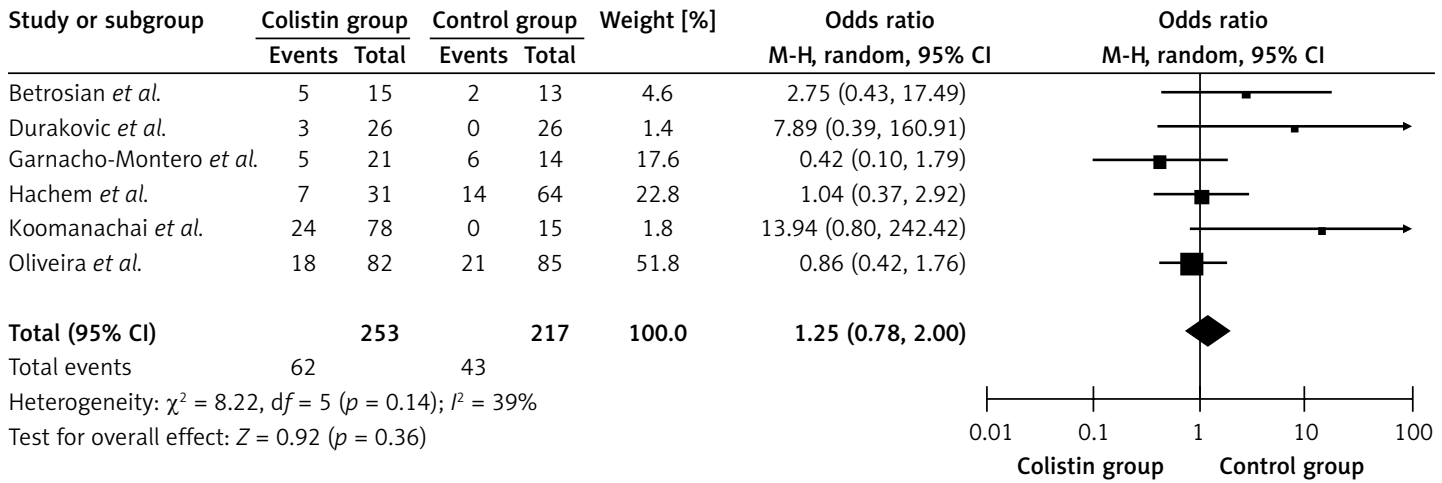

Figure 4. Forest plot of studies with renal damage of colistin group versus control group

el for the remainder of the studies to determine the effect on each overall estimate. The results showed that none of the individual studies substantially influenced the pooled ORs, which implied that our results were statistically reliable.

\section{Discussion}

In the present meta-analyses, we retrieved 9 studies focused on the efficacy and safety of colistin treatment in the patients with pulmonary infection caused by $P$. aeruginosa or A. baumannii. We found that no significant difference in efficacy, mortality and renal damage was detected between colistin and other effective antibiotics. The combined estimate of our analysis was strong across multiple sensitivity analyses and without significant publication bias.

Due to its unsatisfactory efficacy and significant nephrotoxicity and neurotoxicity, use of colistin was limited in clinical practice $[13,33]$. How- 
ever, colistin recently re-entered clinical use with the increasing emergence of MDR Gram-negative bacteria in hospitals $[7,8,15]$. Thus, it is necessary to re-assess the efficacy and safety of colistin treatment. However, the results were inconsistent among previous studies due to small sample sizes or low statistical power. For example, Oliveira et al. reported that ampicillin/sulbactam appears to be a more efficacious therapy than colistin, while Betrosian et al. suggested that colistin and ampicillin/sulbactam were comparably safe and effective treatments. We combined 9 studies and found that compared to other effective agents, colistin showed no significant difference in efficacy when treating pulmonary infection caused by MDR $P$. aeruginosa or $A$. baumannii.

The degree of heterogeneity is one of the major concerns in meta-analysis for the validity of the meta-analysis [34, 35], as non-homogeneous data are liable to result in misleading results. Significant heterogeneity was detected when comparing the efficacy of colistin treatment. To investigate whether the sample size, research year, participants' geographic location (ethnicity) or study method influenced the result, we also conducted a subgroup meta-analysis. We found that the heterogeneity between studies was decreased after stratifying the samples according to their different subgroups. For American samples, significant heterogeneity was observed under the recessive model, while no statistically significant heterogeneity was observed under any model in European samples. The results indicated that ethnicity might play an important role in genetic heterogeneity of patients with pulmonary infection caused by $P$. aeruginosa or $A$. baumannii. The subgroup analysis of clinical response also showed that there was no significant difference between colistin and control groups, suggesting that colistin and other antibiotic therapies are equally efficacious. Asia and Africa as participants' geographic location were not included in the subgroup analyses, due to there being only one study in each region. In addition, dosage and ways of application of drugs were not investigated using the subgroup meta-analysis because these data cannot be extracted in all literatures.

Safety of colistin treatment was also compared with that of other antibiotic therapies using overall meta-analysis. We found that neither hospital mortality nor renal damage was affected by the treatment with colistin or other effective agents, suggesting that colistin also acts as a safe drug in the treatment of pulmonary infection. Actually, colistin is a concentration-dependent drug [36, 37]. In clinical practice, the dosage of colistin was adjusted according to the level of serum creatinine that represents renal function [32]. Thus, no significant renal toxicity of colistin could be explained.
Publication bias is another important factor affecting the quality of meta-analysis. Meta-analyses are prone to publication bias, the problem of selective publication of studies with positive results [38]. The result of Egger's test demonstrated that there was no publication bias in this study (all $p$ values $>0.05$ ). Moreover, the result of sensitivity analysis by removing one study each time and rerunning the model to determine the effect on each overall estimate implied that our results were statistically reliable.

Some limitations of this study should be discussed. First of all, only published studies were included in the present meta-analysis. Thus, publication bias may have occurred, although the use of a statistical test did not show it. Secondly, significant between-study heterogeneity was detected in the current meta-analysis, and may be distorting the meta-analysis. However, it was not a major problem because we did subgroup analysis to reduce the heterogeneity. Meanwhile, different populations may contribute to the heterogeneity. These results should be interpreted with caution because the population from each country was not uniform. Finally, considering that recruited studies were non-RCT and the number of studies was small, more high-quality RCTs are needed to test and verify the results of this meta-analysis. Therefore, we minimized the likelihood of bias by developing a detailed protocol before initiating the study, performing a meticulous search for published studies and using explicit methods for study selection, data extraction and data analysis.

In conclusion, our findings suggest that colistin is a safe and effective drug and could be used as an alternative agent in patients with pulmonary infection caused by MDR $P$. aeruginosa or A. baumannii.

\section{Acknowledgments}

We would like to thank all respondents of the study and all the people who gave help for this study.

\section{Conflict of interest}

The authors declare no conflict of interest.

\section{References}

1. Hachem RY, Chemaly RF, Ahmar CA, et al. Colistin is effective in treatment of infections caused by multidrug-resistant Pseudomonas aeruginosa in cancer patients. Antimicrob Agents Chemother 2007; 51: 1905-11.

2. Zavascki AP, Carvalhaes CG, Picao RC, Gales AC. Multidrug-resistant Pseudomonas aeruginosa and Acinetobacter baumannii: resistance mechanisms and implications for therapy. Expert Rev Anti Infect Ther 2010; 8: 71-93.

3. Navon-Venezia S, Ben-Ami R, Carmeli Y. Update on Pseudomonas aeruginosa and Acinetobacter baumannii infections in the healthcare setting. Curr Opin Infect Dis 2005; 18: 306-13. 
4. Kuo SC, Lee YT, Yang SP, et al. Eradication of multidrug-resistant Acinetobacter baumannii from the respiratory tract with inhaled colistin methanesulfonate: a matched case-control study. Clin Microbiol Infect 2012; 18: 870-6.

5. Falagas ME, Kasiakou SK. Toxicity of polymyxins: a systematic review of the evidence from old and recent studies. Crit Care 2006; 10: R27.

6. Fielding BC, Mnabisa A, Gouws PA, Morris T. Antimicrobial-resistant Klebsiella species isolated from free-range chicken samples in an informal settlement. Arch Med Sci 2012; 8: 39-42.

7. Falagas ME, Kasiakou SK. Colistin: the revival of polymyxins for the management of multidrug-resistant gram-negative bacterial infections. Clin Infect Dis 2005; 40: 1333-41.

8. Li J, Nation RL, Turnidge JD, et al. Colistin: the re-emerging antibiotic for multidrug-resistant Gram-negative bacterial infections. Lancet Infect Dis 2006; 6: 589-601.

9. Catchpole CR, Andrews JM, Brenwald N, Wise R. A reassessment of the in-vitro activity of colistin sulphomethate sodium. J Antimicrob Chemother 1997; 39: 255-60.

10. Evans ME, Feola DJ, Rapp RP. Polymyxin B sulfate and colistin: old antibiotics for emerging multiresistant gram-negative bacteria. Ann Pharmacother 1999; 33: 960-7.

11. Kasiakou SK, Michalopoulos A, Soteriades ES, Samonis G, Sermaides GJ, Falagas ME. Combination therapy with intravenous colistin for management of infections due to multidrug-resistant Gram-negative bacteria in patients without cystic fibrosis. Antimicrob Agents Chemother 2005; 49: 3136-46.

12. Conway SP, Pond MN, Watson A, Etherington C, Robey HL, Goldman MH. Intravenous colistin sulphomethate in acute respiratory exacerbations in adult patients with cystic fibrosis. Thorax 1997; 52: 987-93.

13. Hamer DH. Treatment of nosocomial pneumonia and tracheobronchitis caused by multidrug-resistant Pseudomonas aeruginosa with aerosolized colistin. Am J Respir Crit Care Med 2000; 162: 328-30.

14. Motaouakkil S, Charra B, Hachimi A, et al. Colistin and rifampicin in the treatment of nosocomial infections from multiresistant Acinetobacter baumannii. J Infect 2006; 53: 274-8.

15. Paul M, Bishara J, Levcovich A, et al. Effectiveness and safety of colistin: prospective comparative cohort study. J Antimicrob Chemother 2010; 65: 1019-27.

16. Durakovic N, Radojcic V, Boban A, et al. Efficacy and safety of colistin in the treatment of infections caused by multidrug-resistant Pseudomonas aeruginosa in $\mathrm{pa}$ tients with hematologic malignancy: a matched pair analysis. Intern Med 2011; 50: 1009-13.

17. Gounden R, Bamford C, van Zyl-Smit R, Cohen K, Maartens G. Safety and effectiveness of colistin compared with tobramycin for multi-drug resistant Acinetobacter baumannii infections. BMC Infect Dis 2009; 9: 26.

18. Bergen PJ, Forrest A, Bulitta JB, et al. Clinically relevant plasma concentrations of colistin in combination with imipenem enhance pharmacodynamic activity against multidrug-resistant Pseudomonas aeruginosa at multiple inocula. Antimicrob Agents Chemother 2011; 55 5134-42.

19. Munafo MR, Flint J. Meta-analysis of genetic association studies. Trends in Genetics 2004; 20: 439-44.

20. Wu G, Sun G, Zhao R, Sun M. Clinical outcomes of second-versus first-generation drug-eluting stents in patients with acute myocardial infarction: a meta-analysis of randomized controlled trials. Arch Med Sci 2014; 10: 643-50.

21. Mozaffari S, Nikfar S, Abdollahi M. Efficacy and tolerability of renzapride in irritable bowel syndrome: a meta-analysis of randomized, controlled clinical trials including 2528 patients. Arch Med Sci 2014; 10: 10-8.

22. Deeks JJ, Altman DG, Bradburn MJ. Statistical methods for examining heterogeneity and combining results from several studies in meta-analysis. Systematic Reviews in Health Care: Meta-Analysis in Context Second Edition 2001; 285-312

23. Higgins JP, Thompson SG, Deeks JJ, Altman DG. Measuring inconsistency in meta-analyses. BMJ 2003; 327: 557-60.

24. Mantel N, Haenszel W. Statistical aspects of the analysis of data from retrospective studies of disease. J Natl Cancer Inst 1959; 22: 719-48.

25. DerSimonian R, Laird N. Meta-analysis in clinical trials. Control Clin Trials 1986; 7: 177-88.

26. Egger M, Smith GD, Schneider M, Minder C. Bias in meta-analysis detected by a simple, graphical test. BMJ 1997; 315: 629-34.

27. Betrosian AP, Frantzeskaki F, Xanthaki A, Douzinas EE. Efficacy and safety of high-dose ampicillin/sulbactam vs. colistin as monotherapy for the treatment of multidrug resistant $\langle\mathrm{i}\rangle$ Acinetobacter baumannii</i $>$ ventilator-associated pneumonia. J Infect 2008; 56: 432-6.

28. Kallel H, Hergafi L, Bahloul M, et al. Safety and efficacy of colistin compared with imipenem in the treatment of ventilator-associated pneumonia: a matched case-control study. Intensive Care Med 2007; 33: 1162-7.

29. Oliveira M, Prado G, Costa S, Grinbaum R, Levin A. Ampicillin/sulbactam compared with polymyxins for the treatment of infections caused by carbapenem-resistant Acinetobacter spp. J Antimicrob Chemother 2008; 61: 1369-75.

30. Reina R, Estenssoro E, Sáenz G, et al. Safety and efficacy of colistin in Acinetobacter and Pseudomonas infections: a prospective cohort study. Intensive Care Med 2005; 31: 1058-65.

31. Lu Q, Luo R, Bodin L, et al. Efficacy of high-dose nebulized colistin in ventilator-associated Pneumonia caused by multidrug-resistant Pseudomonas aeruginosa and Acinetobacter baumannii. Anesthesiology 2012; 117: 1335-47.

32. Garnacho-Montero J, Ortiz-Leyba C, Jimenez-Jimenez FJ, et al. Treatment of multidrug-resistant Acinetobacter baumannii ventilator-associated pneumonia (VAP) with intravenous colistin: a comparison with imipenem-susceptible VAP. Clin Infect Dis 2003; 36: 1111-8.

33. Koomanachai P, Tiengrim S, Kiratisin P, Thamlikitkul V. Efficacy and safety of colistin (colistimethate sodium) for therapy of infections caused by multidrug-resistant Pseudomonas aeruginosa and Acinetobacter baumannii in Siriraj Hospital, Bangkok, Thailand. Intern J Infect Dis 2007; 11: 402-6.

34. Moreno SG, Sutton AJ, Thompson JR, Ades A, Abrams KR, Cooper NJ. A generalized weighting regression-derived meta-analysis estimator robust to small-study effects and heterogeneity. Statistics in Medicine 2012; 31: 1407-17.

35. Li Y, Zhou C, Zhou X, Li L, Hui R. Pregnancy-associated plasma protein $\mathrm{A}$ predicts adverse vascular events in patients with coronary heart disease: a systematic review and meta-analysis. Arch Med Sci 2013; 9: 389-97.

36. Gunderson BW, Ibrahim KH, Hovde LB, Fromm TL, Reed MD, Rotschafer JC. Synergistic activity of colistin and ceftazidime against multiantibiotic-resistant Pseudo- 
monas aeruginosa in an in vitro pharmacodynamic model. Antimicrob Agents Chemother 2003; 47: 905-9.

37. Li J, Turnidge J, Milne R, Nation RL, Coulthard K. In vitro pharmacodynamic properties of colistin and colistin methanesulfonate against Pseudomonas aeruginosa isolates from patients with cystic fibrosis. Antimicrob Agents Chemother 2001; 45: 781-5.

38. Niemeyer H, Musch J, Pietrowsky R. Publication bias in meta-analyses of the efficacy of psychotherapeutic interventions for schizophrenia. Schizophrenia Research 2012; 138: 103-12. 